\title{
Spatial analysis of tuberculosis in four main ethnic communities in Taiwan during 2005 to 2009
}

\author{
Pui-Jen Tsai \\ Center for General Education, Aletheia University, New Taipei, Taiwan; puijentsai@gmail.com
}

Received 5 September 2011; revised 16 October 2011; accepted 25 October 2011.

\section{ABSTRACT}

The aim of the present study was to assess spatial features of tuberculosis prevalence and their relationships with four main ethnic communities in Taiwan. Methods of spatial analysis were clustering pattern determination (such as global version of Moran's test and local version of $G_{i}^{*}(d)$ statistic), using logistic regression calculations to identify spatial distributions over a contiguous five years and identify significant similarities, discriminant analysis to classify variables, and geographically weighted regression (GWR) to determine the strength of relationships between tuberculosis prevalence and ethnic variables in spatial features. Tuberculosis demonstrated decreasing trends in prevalence in both genders during 2005 to 2009. All results of the global Moran's tests indicated spatial heterogeneity and clusters in the plain and mountainous Aboriginal townships. The $G_{i}{ }^{*}(d)$ statistic calculated z-score outcomes, categorized as clusters or non-clusters, at at $5 \%$ significance level. According to the stepwise Wilks' lambda discriminant analysis, in the Aborigines and Hoklo communities townships with clusters of tuberculosis cases differentiated from townships without cluster cases, to a greater extent than in the other communities. In the GWR models, the explanatory variables demonstrated significant and positive signs of parameter estimates in clusters occurring in plain and mountainous aboriginal townships. The explanatory variables of both the Hoklo and Hakka communities demonstrated significant, but negative, signs of parameter estimates. The Mainlander community did not significantly associate with cluster patterns of tuberculosis in Taiwan. Results indicated that locations of high tuberculosis prevalence closely related to areas containing higher proportions of the Aboriginal commu- nity in Taiwan. This information is relevant for assessment of spatial risk factors, which, in turn, can facilitate the planning of the most advantageous types of health care policies, and implementation of effective health care services.

Keywords: Tuberculosis; Taiwanese Ethnicity; Global Moran's Test; Local $G_{i}^{*}(d)$ Statistic; Logistic Regression; Discriminant Analysis; Geographically Weighted Regression

\section{INTRODUCTION}

Tuberculosis (TB) is one of the world's principal infectious diseases. In 2007, the World Health Organization (WHO) estimated that more than nine million new cases of TB occur globally, with more than half of these new cases occurring in Asia (55\%). Approximately 1.76 million people died of TB worldwide in 2007. In recent years, with increasing numbers of cases of human immunodeficiency virus (HIV) and multidrug resistant tuberculosis (MDR-TB), prevention of TB has posed a significant challenge. TB is an infectious disease, caused by mycobacteria of the $M$. tuberculosis complex ( $M$. tuberculosis, M. bovis, M. africanum, M. microti, $M$. canettii, M. caprae, M. pinnipedii). Transmission occurs via exposure to tubercle bacilli in airborne droplet nuclei produced by a person with infectious TB during coughing, sneezing, singing, or talking. The infectiousness of the person with TB, the susceptibility of those exposed, the duration of exposure, the proximity to the source case, and the efficiency of cabin ventilation are all factors which can influence the risk of infection. Susceptibility to infection and disease increases in immunocompromised persons (such as human immunodeficiency virus-infected persons) and infants and young children (less than five years of age). When TB develops in the human body, it does so in two stages: first, the individual exposed to M. tuberculosis becomes infected, and second, the infected individual develops the disease (active TB). A small minority $(<10 \%)$ of infected individuals 
subsequently develop active disease and most of them do so within five years. The risk of progression to active TB disease is greatest within the first two years after infection. However, latent infection may persist for life $[1,2]$.

Taiwan has engaged in TB prevention for more than 50 years. For nearly a decade, both the incidence and death rates of TB in Taiwan have gradually declined, yet it remains a prominent infectious disease with the largest number of annual confirmed cases and deaths among all notifiable infectious diseases. According to the Taiwan Tuberculosis Control Report (2009), during each year from to 2005 to 2008 the tuberculosis incidence was 16,472 ( 72.5 per 100,000 population), 15,378 (67.4 per 100,000 population), 14,480 (63.2 per 100,000 population), and 14,265 (62.0 per population), respectively. Overall, the incidence decreased by $14.5 \%$. The incidence rates of all forms of TB and smear-positive TB in males were two to three times higher than in females in 2007. Irrespective of gender, incidence rates increased with age. The numbers of deaths caused by TB cause during each year from 2005 to 2008 were 970 (4.3 per 100,000 population), 832 (3.6 per 100,000 population), 783 (3.4 per 100,000 population), and 762 (3.3 per 100,000 population), respectively. Overall, mortalities decreased by $23.3 \%$. However, the decline in the number of new TB cases in Taiwan was less significant than declines occurring in the U.S., Japan, and Singapore [2].

In a 12 month cohort study in 2006 the treatment success rate of all forms of new TB cases was $70.4 \%$, and the death rate was $18.6 \%$. Of all regions, Central Taiwan had the highest treatment success rate $(72.8 \%)$, while the Eastern area had the lowest $(63.0 \%)$. According to the number of smear-positive TB cases in 2006 and the treatment outcomes of 12 months cohort study, the 2009 WHO Tuberculosis Annual Report described the global success rate as $84.3 \%$ (for the 2006 cohort), achieving the Millennium Development Goal (MDG) of $85 \%$ designated by the WHO. In Taiwan, the treatment success rate of smear-positive cases in a 12 month cohort study was $67 \%$. This value was higher than the rates observed in Japan (53\%) and the U.S. (64\%), but lower than in Singapore (84\%), and did not achieve the WHO assigned target [2].

A number of reports have documented that habitation in mountain aboriginal townships and age of 65 years are two factors which closely associate with the incidence and prevalence of tuberculosis in Taiwan [3-7]. However, research on healthcare problems associated with tuberculosis in Taiwanese ethnic people is limited. The present study employs methods of spatial analysis of areal data to determine spatial features related to tuberculosis and the four major Taiwanese communities.

\section{METHODS}

\subsection{Study Area}

The study was conducted within the main island of Taiwan (excluding all islets), which, in 2008, comprised more than 23 million inhabitants living in an area of $36,000 \mathrm{~km}^{2}$. A total of 349 local administrative government areas, including five main urban areas, two seconddary urban areas, 162 rural townships, and 54 plain and mountainous aboriginal townships, were assessed (Figure 1). According to a bulletin from the Ministry of Interior, issued in 2002, urban areas are regions having at least one metropolitan centre and can include neighbouring cities and townships which share socioeconomic activities. Main urban areas are defined as those with a population larger than one million; specifically, TaipeiKeelung, Kaohsiung, Taichung-Changhua, JhongliTaoyuan, and Tainan. Secondary urban areas are defined as those with a residential population ranging from 0.3 to 1 million (Hsinchu and Chiayi) [8].

\subsection{Data Collection and Management}

The Taiwan National Health Insurance (NHI) program was initiated in 1995 . The coverage rate of the program increased from $92.41 \%$ in 1995 to more than $96.16 \%$ in 2000 , and then further increased to $98 \%$ after the inclusion of active military forces in 2001. At the beginning of 2004, NHI data related to medical care, such as the leading causes of death, were reclassified and reprocessed in relation to smaller units or areas (for example, precincts or townships rather than the country as a

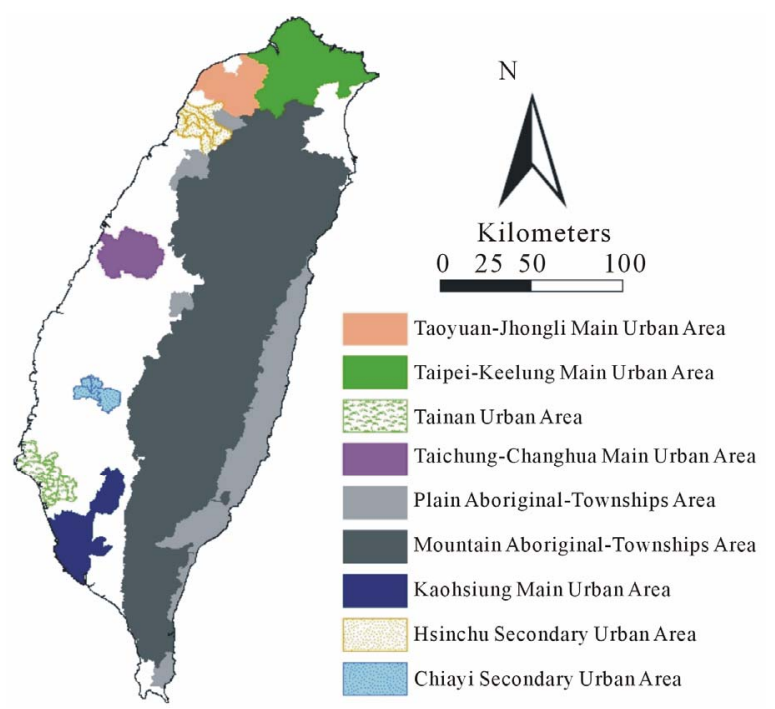

Figure 1. Map of urban regions and aboriginal townships in the study area. Map of the study area divided into 349 administrative districts including 7 urban regions and an integrated area of 54 plain and mountainous aboriginal townships. 
whole). Regional data from the statistical analysis system (SAS) program are announced publicly by the NHI in regular annual reports (for example, NHI, 2005-2009). These reports provide an accurate and reliable data source to researchers for investigation of health care issues in Taiwan [9-13]. The data were collected from contractual medical care institutions, which, in the present study, are institutions where the NHI covers prescription medicinal costs and treatment at outpatient clinics. Such facilities accumulate detailed databases of medical costs for inpatient care. The numbers of outpatient cases were classified in relation to disease codes, as defined in the 1975 edition of "The International Classification of Diseases, 9th Revision, Clinical Modification" (hereafter, ICD 9 CM), 2005-2007 and "The Tenth Revision of the International Statistical Classification of Diseases and Related Health Problems" (hereafter, ICD $10 \mathrm{CM}), 2008-2009$. Criteria for refining the data were first established. However, for calculating medical costs and numbers of visits, the disease code of the first group (main diagnosis code) was used as the cause of disease. Selected data were not included in the final statistical data set, such as cases where patients suffered from diseases which defied code classification, or had mismatched ID numbers. Disease codes were classified according to gender and age. Cases sharing the same ID numbers, despite having different diseases, were counted as separate incidences.

Medical care data obtained from the NHI, 2005-2009 reports were examined, and the prevalence rates of tuberculosis (ICD 02, according to ICD $9 \mathrm{CM}$ standards in 2005-2007 and the disease code of tuberculosis A15A19, classified by ICD 10 CM in 2008-2009) were calculated. The Ministry of the Interior provided the demographic information (the mid-year population of each township) [8]. The age-adjusted standard prevalence rates were calculated with a direct adjustment using the world population in 2000 as the standard population [14]. The age-adjusted standard prevalence rates during 20052009 were calculated according to which of the five year prevalence rates were weighted by persons each year and (or) by persons each gender. The results provided tuberculosis prevalence rates for males and females in the whole of Taiwan and in each township in the study area, and these were consequently applied to spatial autocorrelation analysis.

The percentages of the four major Taiwanese ethnic communities in each township were obtained from an official report of the Council for Hakka Affairs (2004) [15]. According to self-reports in official governmental statistics, Han Chinese constitute 98\% of Taiwan's population, while Taiwanese Aborigines constitute the remaining $2 \%$. The composite category of "Taiwanese people" is often reputed to include a significant population of at least four constituent ethnic groups: the Hoklo $(73.3 \%)$, the Hakka (13.5\%), the Mainlander $(8 \%)$, and the Taiwanese Aborigines (1.9\%) [15]. Figure 2 maps the proportions of ethnic communities in populations of each of the 349 townships.

\subsection{Global Moran's I Statistic}

The global spatial autocorrelation statistical method was used to evaluate correlation between neighbouring observations, and to identify patterns and levels of spatial clustering in neighbouring districts [16]. The Moran's I statistic, similar to the Pearson correlation coefficient [17], is calculated using:

$$
I=\frac{N}{S_{O}} \sum_{i} \sum_{j} w_{i j} \frac{\left(x_{i}-u\right)\left(x_{j}-u\right)}{\sum_{i}\left(x_{i}-u\right)^{2}}
$$

where $N$ is the number of districts and $w_{i j}$ is the element in the spatial weight matrix corresponding to the observation pair $i, j . x_{i}$ and $x_{j}$ are observations for areas $i$ and $j$

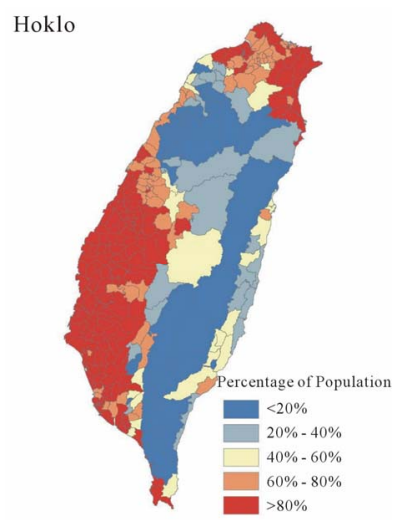

(a)

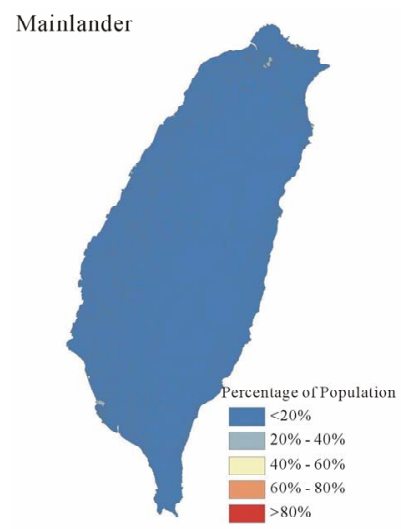

(c)

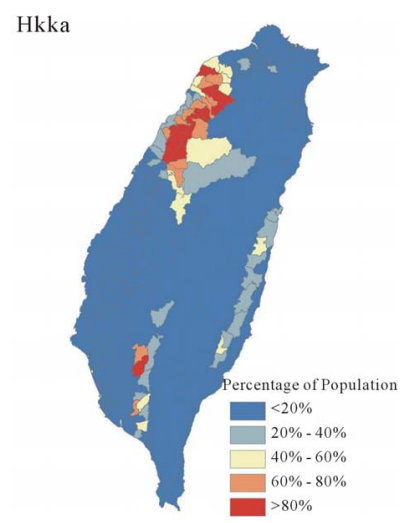

(b)

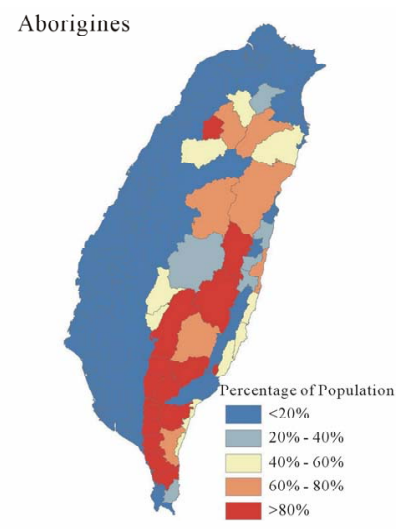

(d)
Figure 2. Maps of population percentages of four Taiwanese ethnic communities in the study area. (a) Hoklo community. (b) Hakka community. (c) Mainlander community. (d) Aboriginal community. 
with mean $u$ and

$$
S_{O}=\sum_{i} \sum_{j} w_{i j}
$$

Since the weights are row-standardized and $\sum w_{i j}=1$, the first step in the spatial autocorrelation analysis was to construct a spatial weight matrix which contained information about the neighbourhood structure for each location. Adjacency was defined as immediately neighboring administrative districts, inclusive of the district itself. Non-neighbouring administrative districts were assigned a weight of zero.

Spatial contiguity for polygons is the property of sharing a common boundary or vertex. Contiguity analysis is an important method for assessing unusual features in the connectivity distribution $[18,19]$. The Queen's measure of contiguity can be utilized to make up for spatial contiguity by incorporating both the Rook and Bishop relationships into a single measure [19].

The administrative districts considered in this study were highly irregular in both shape and size. Tsai et al. (2009) demonstrated that the most appropriate method for quantifying the spatial weights matrix for analysis of connectivity is the first order queen polygon continuity method [7]. The spatial weights/connectivity matrices were utilized in local $G_{i}{ }^{*}(d)$ calculations.

\subsection{Local $G_{i}^{*}(d)$ Statistic}

The local $G_{i}^{*}(d)$ statistic (local G-statistic) is used to determine the statistical significance of local clusters, and to evaluate the spatial extent of these clusters [20,21]. The local G-statistic is useful for identifying individual members of local clusters by determining the spatial dependence and relative magnitudes between an observation and neighboring observations [22]. The local G-statistic can be written as follows $[20,23,24]$ :

$$
G_{i}^{*}(d)=\frac{\sum_{j} w_{i j}(d) x_{j}-W_{i} \bar{x}}{s \sqrt{\frac{\left(n S_{1 i}-W_{i}^{2}\right)}{(n-1)}}} \text {, for all } j
$$

where $x$ is a measure of the prevalence rate of tuberculosis within a given polygon (each administrative district), $w_{i j}$ is a spatial weight which defines neighbouring administrative districts $j$ to $i, W_{i}$ is the sum of the weights $w_{i j}, \quad \bar{x}=\frac{1}{n} \sum_{j} x_{j}, \quad S_{1 i}=\sum_{j} w_{i j}^{2}, s^{2}=\frac{1}{n} \sum_{j} x_{j}^{2}-\bar{x}^{2}$.

Developing the spatial weight $w_{i j}$ is the first step to calculating $G_{i}{ }^{*}(d)$. The spatial weight matrix includes $w_{i j}$ $=1$. In the present study, adjacency was defined using a first order queen polygon continuity weight file which had been constructed based on the districts which share common boundaries and vertices.

Non-neighbouring administrative districts were as- signed a weight of zero. The neighbours of an administrative district were defined as those with which the administrative district shares a boundary. This formed a simple $0 / 1$ matrix; 1 indicated that the municipalities share a common border or vertex, 0 otherwise [23,25].

The local G-statistic included the value in the calculation at $i$. Assuming that $G_{i}^{*}(d)$ is approximately normally distributed [20], the output of $G_{i}^{*}(d)$ can be calculated as a standard normal variant with an associated probability from the z-score distribution [26]. Clusters with a $95 \%$ significance level from a two-tailed normal distribution indicated significant spatial clustering, but only positively significant clusters (z-score values greater than +1.96 ) were mapped.

\subsection{To Determine Space-Time Similarities Using Logistic Regression Model}

Similarities between spatial distribution patterns for a time course during 2005 to 2009 were determined using logistic regression model. The binary response indicated if there was significant autocorrelation between administrative districts or areas. If the absolute value of the z-score of the local $G$-statistics was larger than 1.96 this indicated higher correlation; lower correlation if otherwise. Year was considered an explanatory variable in the logistic regression model. Thus, the model could be expressed as:

$$
\log \left(\frac{\operatorname{Pr}(\text { Higher correlation })}{\operatorname{Pr}(\text { Lower correlation })}\right)=\beta_{0}+\beta_{1} \times \text { Year }
$$

where $\beta_{0}$ and $\beta_{1}$ are the logistic regression coefficients of the model. $\operatorname{Pr}$ (Higher correlation) and $\mathrm{Pr}$ (Lower correlation) denote the "Higher" and "Lower" correlation probabilities, respectively.

\subsection{Discriminant Analysis}

Discriminant analysis is useful for determining which variables discriminate between two or more groups, building a predictive model of group membership based on observed characteristics of each case [27]. It provides a multiple regression equation(s) and those variables which contribute most to the discrimination of group membership are the ones with the largest standardized coefficients. Discrimination analysis is less flexible than regression because it requires the explanatory variables to be normally distributed without equal variance within each group [28]. Using the discriminant analysis, Taiwanese ethnic variables could be identified in the study area. A stepwise Wilks' lamda discriminant analysis was applied to compare tuberculosis prevalence (2005 to 2009) within the townships of $G_{i}{ }^{*}(d)$ test's clusters to those classified as non-clustered relative to four Tai- 
wanese ethnic factors. Clusters were assigned the number 2 if the absolute value of the z-score of the local $G$-statistics was larger than 1.96; non-clusters the number 1 if otherwise.

\subsection{Geographically Weighted Regression}

GWR is an extension of the traditional standard regression framework which allows local, rather than global, parameters to be estimated [29]. It is a type of local statistic which can produce a set of local parameter estimates demonstrating how a relationship varies over space, and then allows examination of the spatial pattern of the local estimates to gain some understanding of possible hidden causes for this pattern [30]. In contrast, a traditional regression method, such as ordinary least squares (OLS), is a type of global statistic which assumes the relationship under study is constant over space, so the parameter is estimated to be the same for the entire study area.

An OLS model can be defined as follows:

$$
y=\beta_{0}+\sum_{i=1}^{p} \beta_{i} X_{i}+\varepsilon
$$

where $\mathrm{y}$ is the response variable, $\beta_{0}$ is the intercept, $\beta_{\mathrm{i}}$ is the parameter estimate (coefficient) for the explanatory variable $\mathrm{x}_{\mathrm{i}}, \mathrm{p}$ is the number of explanatory variables, and $\varepsilon$ is the error term.

The GWR model allows local, rather than global, parameters to be estimated for the study area, and the OLS model can be rewritten as follows:

$$
y_{j}=\beta_{0}\left(u_{j}, v_{j}\right)+\sum_{i-1}^{p} \beta_{i}\left(u_{j}, v_{j}\right) X_{i, j}+\varepsilon_{j}
$$

where $u_{j}$ and $v_{j}$ are the coordinates for each location $\mathrm{j}, \beta_{0}$ $\left(u_{j}, v_{j}\right)$ is the intercept for location $\mathrm{j}$, and $\beta_{i}\left(u_{j}, v_{j}\right)$ is the local parameter estimate for the explanatory variable $\mathrm{x}_{\mathrm{i}}$ at location $\mathrm{j}$.

The weight assigned to each observation is based on a distance decay function centered on observation $i$.

The estimator for the GWR model is similar to the weighted least squares (WLS) global model, except that the weights are conditioned on the location $u$ relative to the observations in the dataset, and hence change for each location. The estimator takes the following form:

$$
\hat{\beta}(u)=\left(X^{T} W(u) X\right)^{-1} X^{T} W(u) y
$$

$W(u)$ is square matrix of weights relative to the position $\mathrm{u}$. A particular location can be indexed $\left(u_{j}, v_{j}\right)$ in the study area. $X^{T} W(u) X$ is the geographically weighted variance-covariance matrix, and $y$ is the vector of the value of the response variable.

The $W(u)$ matrix contains the geographical weights in its leading diagonal and zero in its off-diagonal elements.

$$
\left[\begin{array}{cccc}
w_{1}(u) & 0 & 0 & 0 \\
0 & w_{2}(u) & 0 & 0 \\
0 & 0 & \cdots & 0 \\
0 & 0 & 0 & w_{n}(u)
\end{array}\right]
$$

The distance decay function, which may take a variety of forms, is modified by a bandwidth setting at which distance the weight rapidly approaches zero. In the area in which the present study was conducted, the sample points raised from the polygon centroids were not regularly placed but were clustered. A convenient way of implementing the adaptive bandwidth specification is to select a kernel which allows the same number of sample points for estimations. The weight can be calculated using the specified kernel and setting the value for any observation whose distance is greater than bandwidth to zero. The bisquare function is as follows:

$$
w_{i}\left(u_{j}, v_{j}\right)=\left(1-\left(d_{i}\left(u_{j}, v_{j}\right) / h\right)^{2}\right)^{2}
$$

where $w_{i}\left(u_{j}, v_{j}\right)$ is zero when $d_{i}\left(v_{j}, u_{j}\right)>h . h$ is a quantity known as the bandwidth. This is a near-Guassian function with the useful property of the weight being zero at a finite distance.

The bandwidth was chosen by minimizing the akaike information criterion (AIC) score, defined as, calculated using:

$$
A I C_{c}=2 n \log _{c}(\hat{\sigma})+n \log _{c}(2 \pi)+n\left\{\frac{n+\operatorname{tr}(S)}{n-2-\operatorname{tr}(S)}\right\}
$$

where $\operatorname{tr}(S)$ is the trace of the hat matrix. The AIC method has the advantage of taking into account the fact that the degrees of freedom may vary among models centered on different observations. The optimal bandwidth was determined by minimizing the corrected AIC, as described in Fotheringham et al., 2002. GWR models produce a set of local regression results, including local parameter estimates and the local residuals, which can all be mapped to demonstrate their spatial variability.

The Benjamini-Hochberg $(\mathrm{B}-\mathrm{H})$ procedure is manipulated to control the false discovery rate, which modifies the significance level for each separate test consistently. In the present study, it was used as a solution to determine the significance of parameter estimates raised from the GWR model. Thissen et al. (2002) reported a quick and easy method for calculating the B-H procedure false discovery rate using Microsoft Excel [31]. The B-H approach achieves control of the FDR by sequentially comparing the observed $p$ value for each of a family of multiple test statistics, in order from largest to smallest, 
to a list of computed B-H critical values $[p B-H(i)]$. The critical value on the list is determined for each test statistic, indexed by $i$, by linear interpolation between $\alpha / 2$ (for the largest observed $p$ value) to $(\alpha / 2) / m$, where $m$ is the family size (for the smallest of the $p$ values). The last value is the Bonferroni critical value, so the reason for the gain in power of $\mathrm{B}-\mathrm{H}$ relative to Bonferroni is clear; In the B-H approach, only the smallest of the $m$ observed $p$ values is compared to the Bonferroni critical value. All of the other $p$ values are calculated to less stringent criteria [31]. The local parameter is estimated to be signifycant if the $p$ value is less than the $\mathrm{B}-\mathrm{H}$ critical value; otherwise it is deemed non-significant.

Global Moran's I statistic, local $G_{i}^{*}(d)$ statistic and geographically weighted regression were employed and mapped using ArcMap 9.3. SPSS12 was used to develop logistic regression models for testing space-time similarities and conducting discriminant analysis.

\section{RESULTS}

Table 1 summarizes the tuberculosis prevalence rates In Taiwan during 2005 to 2009. Both genders demonstrated declining trends in tuberculosis prevalence during the evaluation period.

Table 2 summarizes the global autocorrelation statistical findings for tuberculosis in Taiwan during each of the five years from 2005 to 2009. All the results of the global Moran's tests are statistically significant (z-scores greater than 1.96) and indicate spatial heterogeneity.

Table 1. Prevalence of tuberculosis according to gender in Taiwan during 2005 to 2009 .

\begin{tabular}{ccc}
\hline Year & Male $^{*}$ & Female $^{*}$ \\
\hline 2005 & 373.8 & 206.6 \\
2006 & 351.3 & 192.6 \\
2007 & 296.1 & 163.6 \\
2008 & 247.2 & 136 \\
2009 & 223.4 & 125.6 \\
\hline
\end{tabular}

*indicates the prevalence per 100,000 people.

Table 2. Global autocorrelation analysis of tuberculosis in Taiwan during 2005 to 2009 .

\begin{tabular}{ccc}
\hline Year & Moran's Index & Z(I) \\
\hline 2005 & 0.59 & 18.7 \\
2006 & 0.59 & 18.97 \\
2007 & 0.58 & 18.68 \\
2008 & 0.51 & 17.02 \\
2009 & 0.54 & 17.5 \\
\hline
\end{tabular}

$\mathrm{Z}(\mathrm{I})$ : a value greater than 1.96 is considered statistically significant.
Figure 3 displays the spatial clusters (hot spots) for tuberculosis in Taiwan in each of the five years from 2005 to 2009 , obtained using the local $G_{i}{ }^{*}(d)$ statistic. The z-score outcomes calculated by the $G_{i}^{*}(d)$ statistic are categorized as clusters or non-clusters at a $5 \%$ significance level. Clusters are located in the plain and mountainous Aboriginal townships.

Table 3 displays the result from the logistic regression model, used to determine the similarity of spatial patterns of tuberculosis during 2005 to 2009. This result indicates the acceptance of the null hypothesis (a $p$ value greater than 0.05) and that all cases of tuberculosis related to spatial patterns are similar within a contiguous five years period (2005 to 2009). Therefore, the prevalence rates of TB in each township, as weighted by proportions of the population per year (during 2005 to 2009), were used to fit the GWR models.

Table 4 displays the ethnic variables remaining after the discriminant analysis, their coefficients, eigenvalue, grouping accuracy, and the numbers of clusters of townships with and without clusters of tuberculosis cases.

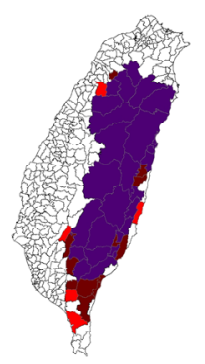

(a)

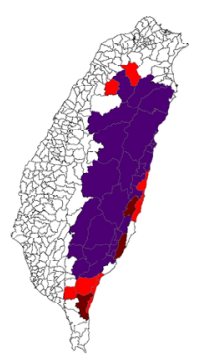

(d)

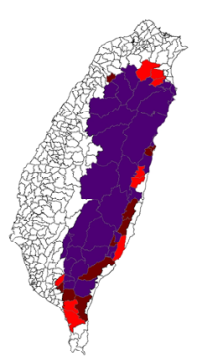

(b)

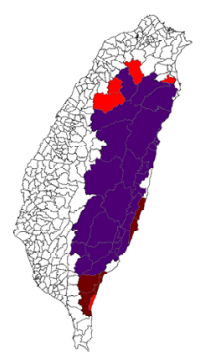

(e)

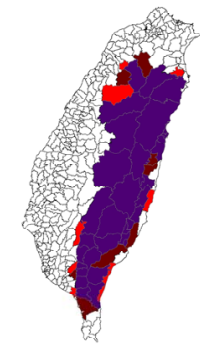

(c)
Figure 3. Spatial clusters (hotspots) of tuberculosis in Taiwan during 2005 to 2009. (a): 2005. (b): 2006. (c): 2007. (d): 2008. (e): 2009 .

Table 3. Space-time similarities of tuberculosis in Taiwan during 2005 to 2009.

\begin{tabular}{ccc}
\hline Disease & $p$ value & Description \\
\hline Tuberculosis & 0.279 & similarity \\
\hline
\end{tabular}

A $p$ value lower than 1.96 is considered statistically non-significant and indicates similarity over a contiguous five year period. 
According to the results of the step-wise Wilks' lambda discriminant analysis, in the Aboriginal and Hoklo communities, townships with clusters of tuberculosis cases differentiated from those without clusters of tuberculosis cases, to a greater extent than in other communities.

Figures 4 to 7 present maps of parameter estimates, the significant determination of the false discovery rate, and local $\mathrm{R}^{2}$, in which tuberculosis figures fit the GWR models with the explanatory variables of the Hoklo, the Hakka, the Mainlander, and the Aboriginal communities, respectively. In the GWR models, the explanatory variables of the Aborigines displayed significant and positive

Table 4. Taiwanese ethnic communities following discriminant analysis.

\begin{tabular}{|c|c|c|c|c|c|}
\hline Year & Variable & Classification function coefficient & Eigen value & Clusters/non-clusters & Grouping accuracy \\
\hline \multirow[t]{3}{*}{$2005-2009$} & Hoklo & -0.09 & 0.861 & $42 / 307$ & $46.24 \%$ \\
\hline & Aborigines & 0.054 & & & \\
\hline & Constant & 0.177 & & & \\
\hline
\end{tabular}

Taiwanese ethnic communities, their coefficients, eigenvalue, the number of census townships with and without tuberculosis clusters, and grouping accuracy.

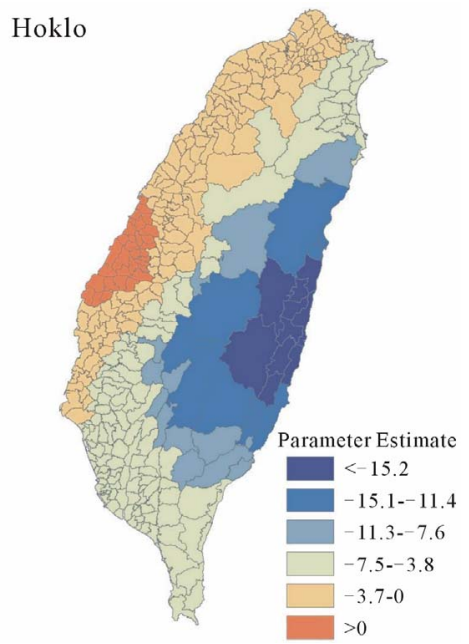

(a)

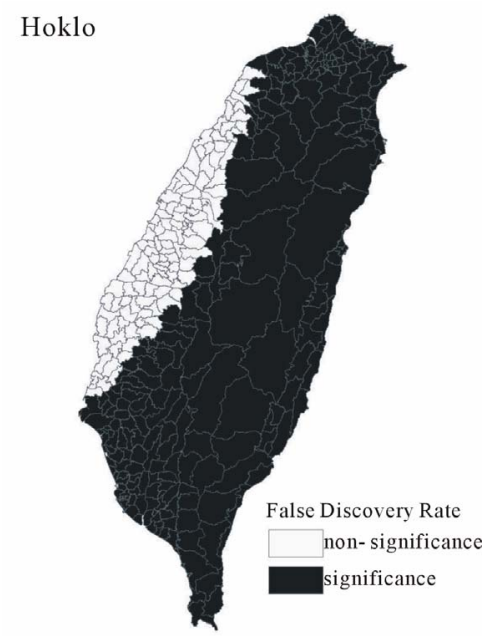

(b)

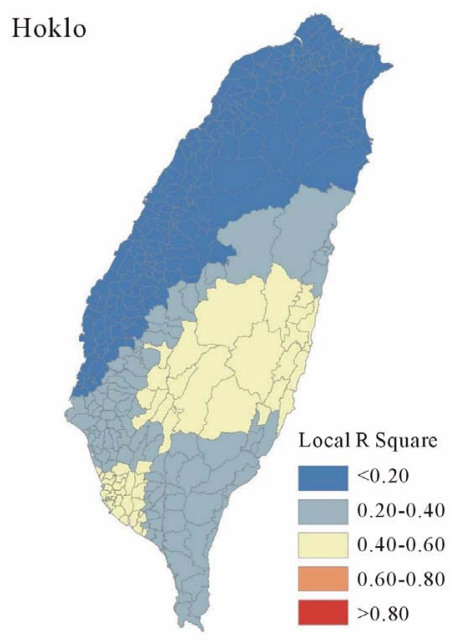

(c)

Figure 4. Results of the GWR model for tuberculosis and percentage of the Hoklo community. (a) shows the parameter estimate. (b) shows the false discovery rate. (c) shows the local R square.

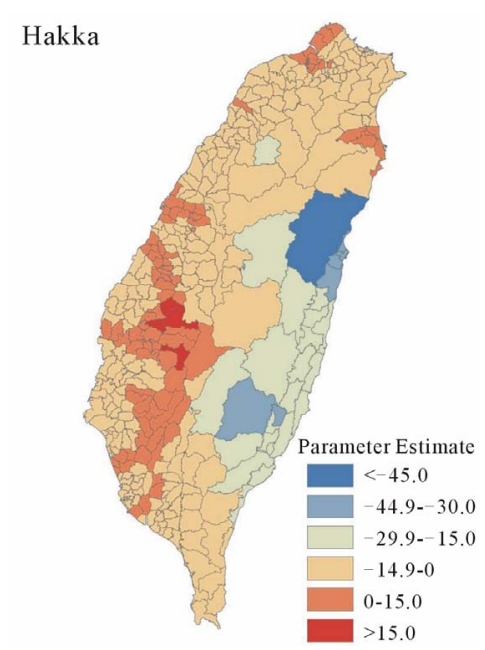

(a)

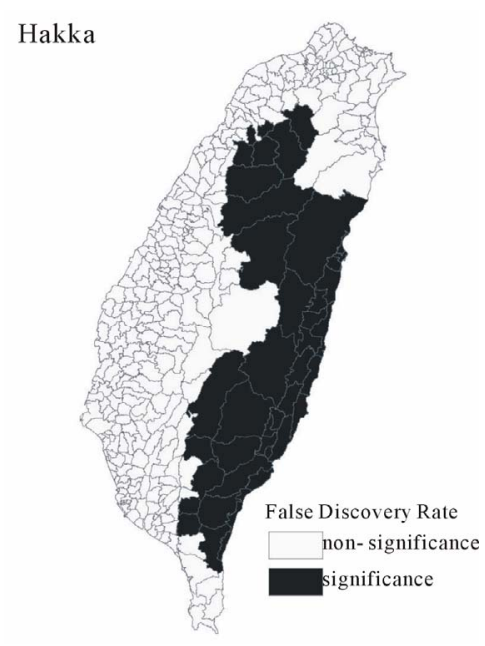

(b)

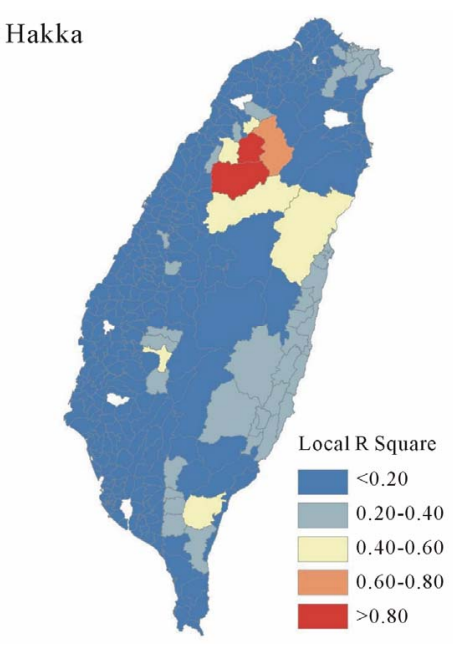

(c)

Figure 5. Results of the GWR model for tuberculosis and percentage of the Hakka community. (a) shows the parameter estimate. (b) shows the false discovery rate. (c) shows the local $\mathrm{R}$ square. 


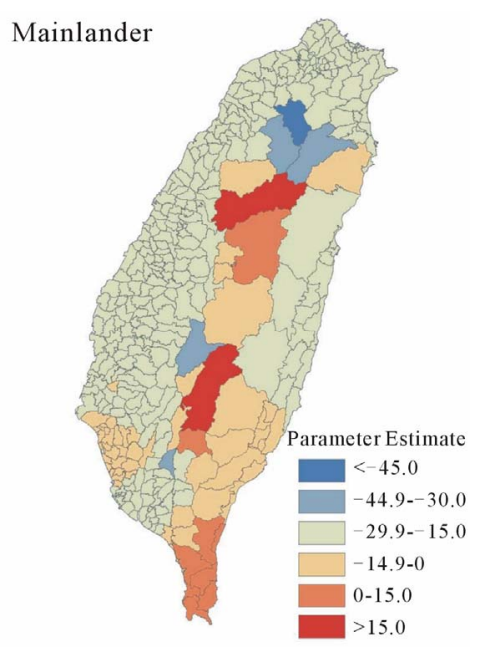

(a)

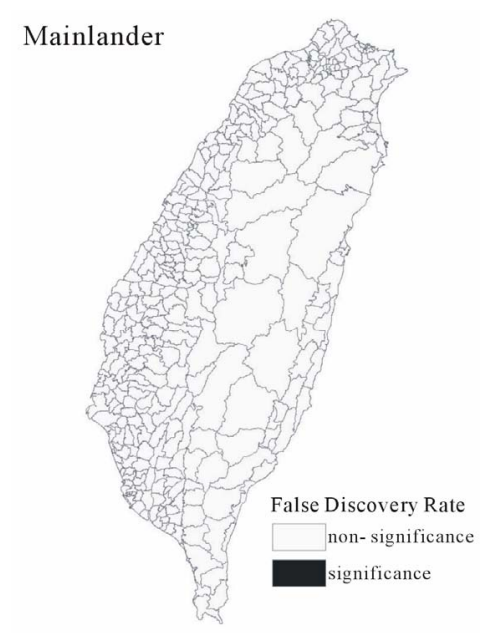

(b)

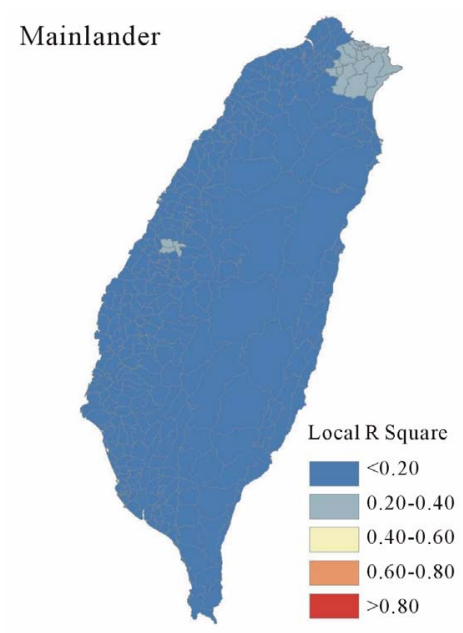

(c)

Figure 6. Results of the GWR model for tuberculosis and percentage of the Mainlander community. (a) shows the parameter estimate. (b) shows the false discovery rate. (c) shows the local R square.

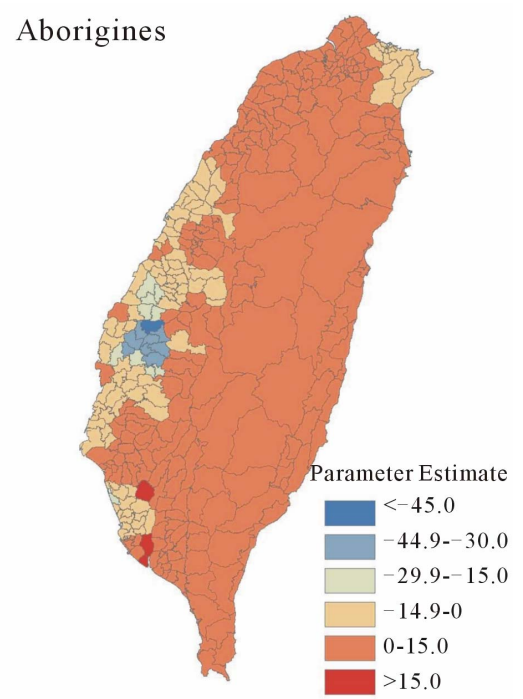

(a)

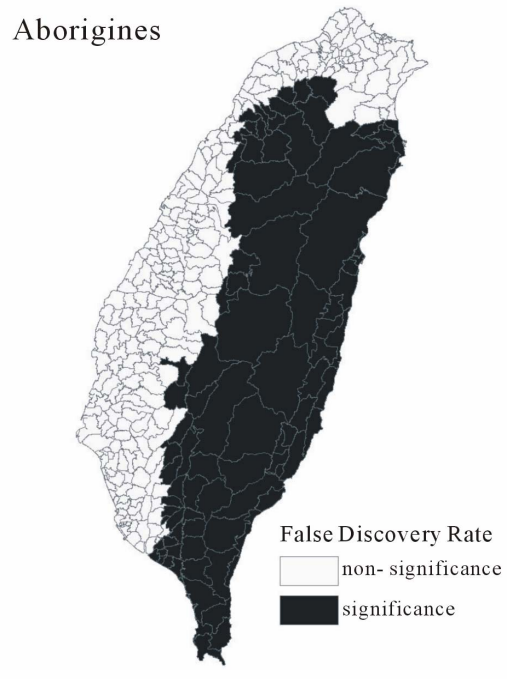

(b)

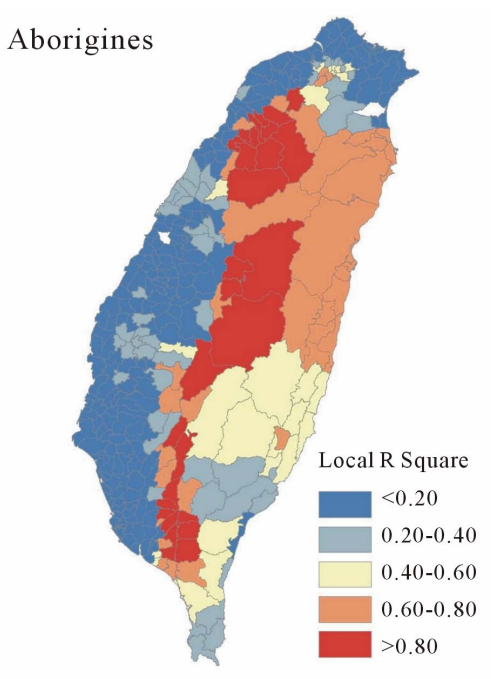

(c)

Figure 7. Results of the GWR model for tuberculosis and percentage of the Aboriginal community. (a) shows the parameter estimate. (b) shows the false discovery rate. (c) shows the local $\mathrm{R}$ square.

signs of parameter estimates in clusters of plain and mountainous aboriginal townships. The explanatory variables of the Hoklo and Hakka communities displayed significant, but negative, signs of parameter estimates. The Mainlander community did not signifycantly associate with the cluster patterns of tuberculosis in Taiwan.

\section{DISCUSSION}

Discriminant analysis is a technique used to determine which variables discriminate between two or more groups, building a predictive model of group membership based on observed characteristics of each case $[27,32]$. It pro- vides a multiple regression equation(s) and those variables which contribute most to the discrimination of group membership are the ones with the largest standardized coefficients.

Stepwise discriminant analysis, like its parallel multiple regression, provides a method of determining the best set of explanatory variables. Used in an exploratory situation it can identify those variables from a larger number of studied variables. In the present study, the stepwise Wilks' lambda discriminant analysis differentiated townships with clusters of tuberculosis cases from those without cluster cases. The results indicated that, in 
the Aboriginal and Hoklo communities, townships with clusters of tuberculosis cases differentiated from those without cluster cases to a greater extent than in other communities. However, this analysis could only provide the best set of explanatory variables in global version.

GWR is a local version of spatial regression which generates parameters disaggregated by the spatial units of analysis. This allows the assessment of spatial heterogeneity in the estimated relationships between the response and explanatory variables. In the global model, it is usual to test whether the parameter (referred to as the coefficient) estimates significantly differ from zero. This can be accomplished with a t-test, in which the output provides the $\mathrm{t}$ statistics and their associated $p$ values. A parameter may have a value estimated as little more than zero; therefore associated with a variable whose variation does not contribute to the model. The model excludes variables with non-significant parameter estimates. In the GWR, one set of parameters associates with each regression point, as well as one set of standard errors, therefore potentially hundreds or thousands of tests would be required to determine if parameters are locally significant. Parameter estimates for variables close to zero often have a tendency for spatial clustering, indicating that in these parts of the study area, changes in this variable do not influence changes in the response variable [33]. The Benjamini-Hochberg (1995) False Discovery Rate (FDR) procedure represents solution for GWR, modifying the significance level for each separate test in a consistent manner $[33,34]$. According to data from the Center for Disease Control in Taiwan, there is a four-fold higher incidence of tuberculosis in aboriginal portions of the population than in people of Han Chinese ethnicity (Hans), consistingof Hoklo, Hakka and Mainlander communities [4]. Analysts have assigned blame to environmental factors, including hygiene, income, and social behavior (such as alcoholism) for the prevalence of tuberculosis in aboriginal populations. Genetic variations in NRAMP 1 may also affect susceptibility to and increase the risk of tuberculosis in Taiwanese aboriginals [35]. GWR models provide more detailed analyses which can estimate relationships between the response and explanatory variables in local version. The present study's findings support the results obtained in previous investigations.

The present study employed methods of spatial analysis to evaluate the strength of relations between spatial distribution of tuberculosis and Taiwanese ethnic communities. Spatial autocorrelation calculations, space-time similarities determined by logistic regression models, variables differentiated by discriminant analysis, and geographically weighted regression (GWR) determined the strength of relations between the prevalence of tu- berculosis and ethnic variables in spatial features. This is relevant to the assessment of spatial risk factors, which, in turn, can facilitate the planning of the most advantageous types of health care policies, and implementation of effective health care services. The study findings indicate that the locations of higher tuberculosis prevalence closely relate to areas with higher proportions of Aboriginal communities in Taiwan.

\section{ACKNOWLEDGEMENTS}

The author wishes to thank Taiwan's Department of Health for providing the National Health Insurance and Centers for Disease Control databases, and the Council for Hakka Affairs for providing Taiwanese ethnicity statistics.

\section{REFERENCES}

[1] World Health Organization (2008) Tuberculosis and air travel: Guidelines for prevention and control. 3rd Edition. World Health Organization press, Geneva.

[2] Centers for Disease Control (2009) Taiwan tuberculosis control report 2009. Centers for Disease Control (Taiwan), Taipei.

[3] Centers for Disease Control (2006) Statistics of communicable diseases and surveillance report, Republic of China 2005. Centers for Disease Control (Taiwan), Taipei.

[4] Centers for Disease Control (2007) Statistics of communicable diseases and surveillance report, Republic of China 2006. Centers for Disease Control (Taiwan), Taipei.

[5] Centers for Disease Control (2008) Statistics of communicable diseases and surveillance report, Republic of China 2007. Centers for Disease Control (Taiwan), Taipei.

[6] Centers for Disease Control (2009) Statistics of communicable diseases and surveillance report, Republic of China 2008. Centers for Disease Control (Taiwan), Taipei.

[7] Tsai, P.J., Lin, M.L., Chu, C.M. and Perng, C.H. (2009) Spatial autocorrelation analysis of health care hotspots in Taiwan in 2006. BMC Public Health, 9, 464.

doi:10.1186/1471-2458-9-464

[8] Ministry of the Interior (2010) The Demographic DataBase. http://www.moi.gov.tw/stat/index.aspx

[9] National Health Insurance (2007) Statistical annual report of medical care 2005. National Health Insurance (Taiwan), Taipei.

[10] National Health Insurance (2008) Statistical annual report of medical care 2006. National Health Insurance (Taiwan), Taipei.

[11] National Health Insurance (2009) Statistical annual report of medical care 2007. National Health Insurance (Taiwan), Taipei.

[12] National Health Insurance (2010) Statistical annual report of medical care 2008. National Health Insurance (Taiwan), Taipei.

[13] National Health Insurance (2011) Statistical annual report of medical care 2009. National Health Insurance (Taiwan), Taipei.

[14] Ahmad, O.E., Boschi-Pinto, C., Lopez, A.D., Murray, 
C.J.L., Lozano, R. and Inoue, M. (2000) Age standardization of rates: A new WHO standard (GPE discussion paper series, No. 31). World Health Organization Press, Geneva.

[15] Council for Hakka Affairs (2009) Hakka Population Census. http://www.hakka.gov.tw

[16] Boots, B.N. and Getis, A. (1998) Point pattern analysis. Sage Publications, Newbury Park.

[17] Cliff, A.C. and Ord, J.K. (1973) Spatial autocorrelation. Pion Limited, London.

[18] Legendre, P. and Legendre, L. (1998) Numerical ecology, 2nd English Edition. Elsevier, Amsterdam.

[19] Grubesic, T.H. (2008) Zip codes and spatial analysis: problems and prospects. Socio-Economic Planning Sciences, 42, 129-149. doi:10.1016/j.seps.2006.09.001

[20] Getis, A. and Ord, J.K. (1992) The analysis of spatial association by use of distance statistics. Geographical Analysis, 24, 189-206. doi:10.1111/j.1538-4632.1992.tb00261.x

[21] Ord, J.K. and Getis, A. (1995) Local spatial autocorrelation statistics: Distributional issues and an application. Geographical Analysis, 27, 286-306. doi:10.1111/i.1538-4632.1995.tb00912.x

[22] Getis, A., Morrison, A.C., Gray, K. and Scott, T.W. (2003) Characteristics of the spatial pattern of the dengue vector, Aedes aegypti, in Iquitos, Peru. American Journal of Tropical Medicine and Hygiene, 69, 494-505.

[23] Wu, J., Wang, J., Meng, B., Chen, G., Pang, L., Song, X., Zhang, K., Zhang, T. and Zheng, X. (2004) Exploratory spa- tial data analysis for the identification of risk factors to birth defects. BMC Public Health, 4, 23. doi:10.1186/1471-2458-4-23

[24] Feser, E., Sweeney, S. and Renski, H. (2005) A descryptive analysis of discrete U.S. industrial complexes. Journal of Regional Science, 45, 395-419. doi:10.1111/j.0022-4146.2005.00376.x

[25] Ceccato, V. and Persson, L.O. (2002) Dynamics of rural areas: An assessment of clusters of employment in Sweden. Journal of Rural Studies, 18, 49-63. doi:10.1016/S0743-0167(01)00028-6

[26] MacKellar, F.L. (1993) Early mortality data: Sources and difficulties of interpretation. In: Kiple, K.F., Ed., The Cambridge World History of Human Disease, Cambridge University Press, Cambridge, 209-213. doi:10.1017/CHOL9780521332866.024

[27] Huberty, C.J. and Olejnik, S. (2006) Applied MANOVA and discriminant analysis. 2nd Edition, John Wiley \& Sons Inc, New York. doi:10.1002/047178947X

[28] Tabachnick, B.G. and Fidell, L.S. (2001) Using multivariate statistics, 4th edition. Allyn \& Bacon, Boston,

[29] Fotheringham, A.S., Brunsdon, C. and Charlton, M. (1998) Geographically weighted regression: A natural evolution of the expansion method for spatial data analysis. Environment and Planning A, 30, 1905-1927. doi:10.1068/a301905

[30] Fotheringham, A.S., Brunsdon, C. and Charlton, M. (2002) Geographically weighted regression: The analysis of spatially varying relationships. John Wiley \& Sons Inc, Chichester.

[31] Thissen, D., Steinberg, L. and Kuang, D. (2002) Quick and easy implemantation of the Benjamini-Hochberg procedure for controlling the false positive rate in multiple comparisons. Journal of Educational and Behavioral Statistics, 27, 77-83. doi:10.3102/10769986027001077

[32] Pfeiffer, D., Robinson, T., Stevenson, M., Rogers, D. and Clements, A. (2008) Identifying factors associated with the spatial distribution of disease. In: Stevenson, M., Rogers, D. and Clements, A., Eds., Spatial Analysis in Epidemiology, Oxford University Press, New York, 103-106. doi:10.1093/acprof:oso/9780198509882.003.0007

[33] Charlton, M., Fotheringham, A.S. (2009) Geographically Weighted Regression white Paper. http://ncg.nuim.ie/ncg/GWR/GWR WhitePaper.pdf

[34] Benjamini, Y. and Hochberg, Y. (1995) Controlling the false discovery rate: A practical and powerful approach to multiple testing. Journal of the Royal Statistical Society Series B, 57, 289-300.

[35] Hsu, Y.H., Chen, C.W., Sun, H.S., Jou, R., Lee, J.J. and Su, I.J. (2006) Association of NRAMP 1 gene polymorphism with susceptibility to tuberculosis in Taiwanese aboriginals. Journal of the Formosan Medical Association, 105, 363-369. doi:10.1016/S0929-6646(09)60131-5 